Published in final edited form as:

Cogn Behav Neurol. 2009 March ; 22(1): 22-27. doi:10.1097/WNN.0b013e318192cc8d.

\title{
Rapidly Progressive Young-Onset Dementia
}

\author{
Brendan J. Kelley, MD, Bradley F. Boeve, MD, and Keith A. Josephs, MD MST \\ Department of Neurology, Mayo Clinic, Rochester, Minnesota; and Robert H. and Clarice Smith and \\ Abigail Van Buren Alzheimer's Disease Research Program of the Mayo Foundation
}

\begin{abstract}
Objective-To characterize a cohort of individuals who have experienced rapidly progressive dementia with onset prior to age 45.

Background-Very little data regarding the clinical features or clinical spectrum of rapidly progressive young-onset dementia (RP-YOD) is available, primarily consisting of case reports or small series.

Methods-A search of the Mayo Clinic medical record was employed to identify patients who had onset prior to age 45 of rapidly progressive dementia. All available medical records, laboratory data, neuroimaging studies, and pathological data were reviewed.
\end{abstract}

Results-Twenty-two patients met the pre-defined inclusion and exclusion criteria. Behavioral and affective disorders, cerebellar dysfunction and visual and/or oculomotor dysfunction were common early clinical features within the cohort, as were clinical features often associated with CreutzfeldtJakob disease (CJD). Diagnostic testing identified an etiology in most patients.

Conclusions-Presentations of RP-YOD result from a variety of etiologies and significant overlap in clinical features is observed. Clinical features often associated with CJD appear to be common within the entire cohort of RP-YOD patients. Diagnostic studies aided in establishing a diagnosis in most patients, however five had uncertain diagnoses despite exhaustive evaluation.

\section{Keywords}

rapidly progressive dementia; young-onset dementia; presenile dementia; Creutzfeldt-Jakob disease; young-onset

\section{Introduction}

Rapidly progressive dementia constitutes a worrisome clinical presentation at any age, perhaps even more so in individuals younger than age 45 . The diagnosis most commonly thought of in the context of rapidly progressive dementia is Creutzfeldt-Jakob disease (CJD), although in truth, the differential diagnosis is broad. ${ }^{1}$ Very little literature exists regarding the clinical features and diagnoses established in younger individuals experiencing rapidly progressive dementia, due primarily to the rarity of presentation.

Patients with rapidly progressive young-onset dementia (RP-YOD) are typically referred for neurologic consultation, and often are eventually referred to academic medical centers. Due to the concerning nature of this presentation, wide differential diagnosis, challenge to establishing diagnoses, and rarity of cases, we sought to examine all cases with RP-YOD evaluated at our

Correspondence to: Keith A. Josephs, M.D. Mayo Clinic Department of Neurology 200 First Street SW Rochester, MN 55905 Phone: 507-538-1038 Fax: 507-538-6012 josephs.keith@ mayo.edu. 
center over a period of time. We were particularly interested in patients who were ambulatory and referred for evaluation in the outpatient setting; those with acute encephalopathy requiring hospitalization were not the focus of this study.

\section{Methods}

This protocol was approved by the Mayo Foundation Institutional Review Board.

\section{Patient identification}

The Mayo Clinic Medical Record Linkage System was searched using relevant Hospital International Classification of Diseases Adapted (HICDA) codes for dementia and neurological disorders which may present with dementia to identify patients age 17 to 45 who were evaluated in the outpatient clinic at Mayo Clinic Rochester from 1996 through 2006 inclusive. All available medical records were reviewed and relevant clinical data extracted. The age at onset was considered to be the age at which cognitive changes were apparent to the patient, family, or close associates.

\section{Inclusion Criteria}

Included patients had onset of rapidly progressive cognitive decline severe enough to impair daily functioning between the ages 17-45 years. We required completion of high-school education as evidence of previously normal cognitive functioning until the age of 17 . Rapid progression was defined as progression to severe dementia or death within 18 months of clinical onset. Severe dementia was taken to mean complete or near-complete inability to perform basic activities of daily living. Although a Clinical Dementia Rating (CDR) assessment was not performed at the time of clinical evaluations, these subjects would be best estimated to have a CDR of two or greater.

\section{Exclusion Criteria}

We excluded patients with longstanding static encephalopathy or mental retardation. Patients requiring special education throughout their academic career were excluded. Those having cognitive deficits referable to a specific event (e.g. traumatic brain injury, stroke, subarachnoid hemorrhage, etc.) were excluded.

\section{Results}

We identified 22 patients (11 female) meeting our predefined inclusion and exclusion criteria. Mean age at onset was 34.1 years (median 37.5 years, range 18-44 years) and mean age at presentation was 34.9 (median 39 years, range 18-44 years). The mean duration from onset to presentation was 7 months (median 4 months, range 1 to 23 months). A family history of earlyonset dementia (prior to age 65) or of a neurological presentation similar to the proband was reported in four (18\%) cases.

Clinical onset was subacute (significant decline occurring within 6 weeks of onset) in 9 and gradual (decline occurring over 2 months or longer) in 13. One individual followed a rapidly progressive course, but subsequently improved after a diagnosis of limbic encephalitis associated with retroperitnoneal teratoma was established and treatment was instituted. Etiologies identified in these 22 patients are indicated, along with ages at onset and presentation, in Table 1. Of note, definite or presumed CJD was the final diagnosis in 7 (32\%).

The early clinical features (present within one month of onset) of this cohort are detailed in Table 1 . These features were reported by the family and/or noted during previous neurological consultation (if this was available) to have been present within one month of onset. The features 
recorded in Table 2 were also noted at the time of presentation. Seven patients exhibited myoclonus, four of whom had CJD. Cerebellar dysfunction was an early feature in nine patients, among whom four had CJD. Parkinsonism was evident at presentation in four individuals, one of whom had CJD. Psychiatric symptoms were present in eleven patients. Three patients manifested psychosis and one other patient experienced visual hallucinations without other psychotic features. Visual/oculomotor abnormalities were demonstrated in seven patients.

Serological testing identified specific abnormalities in one case; previously unknown HIV seropositivity in the patient who presented with progressive multifocal leukoencephalopathy (PML). No specific antibody was identified in the patient presenting with limbic encephalitis.

Cerebrospinal fluid (CSF) analysis was available for review in 18 patients. Routine analysis identified mild protein elevations in eight individuals (mean $63.4 \pm 9.9 \mathrm{mg} / \mathrm{dL}$, range $49-80$ $\mathrm{mg} / \mathrm{dL}$ ) and marked protein elevation $(762 \mathrm{mg} / \mathrm{dL}$ ) with xanthochromia in patient 16 (diagnosis mitochondrial disorder). The CSF level of 14-3-3 protein was tested in five patients. In two of four CJD patients, 14-3-3 protein was elevated and the 14-3-3 protein was equivocal in one individual with unknown etiology. Neuron specific enolase (NSE) was tested in eleven patients. NSE was elevated in four of four tested CJD patients and in one patient with adult onset leukodystrophy with axonal spheroids. CSF JC virus PCR was positive in patient 2 (diagnosis PML).

CSF was collected on all patients, with a mean of 1.7 collections per patient (median 1, range 1-4 collections). None of the repeated routine analyses identified a diagnostic abnormality. Commonly, CSF analysis was repeated to perform non-routine analyses to investigate specific etiologies, although this was successful in only one case.

All patients had one or more brain MRIs during the course of evaluation. Abnormalities were noted on MRI in 19 individuals. Diagnostic neuroimaging findings were identified in seven (32\%) individuals (four with CJD, one with PML, one with Fahr disease, and one with paraneoplastic limbic encephalitis) (see Table 2). Prominent frontotemporal atrophy was noted in the patient with frontotemporal lobar degeneration with motor neuron disease (FTLDMND). In patient 22, diagnosed with mitochondrial disorder, several nondiagnostic MRI abnormalities were noted.

Electroencephalography (EEG) was performed in 19 individuals, and was abnormal in 18 (summarized in Table 2). The most commonly identified abnormality was background slowing (94\%). Periodic sharp wave complexes (PSWCs) were seen in one patient who had CJD (14\% of the CJD cases). Periodic lateralized epileptiform discharges were noted in two individuals (patient 8 with Fahr's disease and patient 22 with paraneoplastic limbic encephalitis) and triphasic waves were identified in patient 16, who had a mitochondrial disorder.

Brain biopsy was performed in 6 patients (27\%) and was diagnostic in 2 (33\%). In patients 8, 1318 and 20, regions of MRI signal abnormality served as biopsy targets. Autopsy data was available for nine patients. Pathological findings are summarized in Table 2. The individual with autopsy-proven FTLD-MND had previously undergone cerebral biopsy, with nondiagnostic findings.

\section{Discussion}

Rapidly progressive dementia is frequently suspected (or even presumed) to represent prion disease. This series provides clinicians with a context in which to evaluate patients having rapidly progressive young onset dementia, emphasizing that disorders other than CJD may cause RP-YOD. Although no specific treatments were available for most patients in this series, 
one patient, whose clinical presentation suggested prion disease, was found to have limbic encephalitis and responded well to treatment. Furthermore, several patients had identifiable errors of metabolism or infectious etiologies, for which specific treatments may become available in the future.

Metabolic disorders are commonly thought to progress insidiously. Metabolic etiologies were identified in $14 \%$ of our cohort, illustrating that these disorders should be considered in the differential diagnosis for RP-YOD. Infectious/prion etiologies were common among this cohort, with CJD accounting for the majority of patients having an infectious/prion etiology, as would be expected. Two patients presented with RP-YOD in association with other degenerative disorders as well. Autoimmune encephalopathy manifesting as rapidly progressive dementia has been previously reported, ${ }^{2}$ but an autoimmune/inflammatory etiology accounted for only one patient in this rapidly-progressive cohort (4.5\%). Steroidresponsive encephalopathy associated with autoimmune thyroiditis, sometimes referred to as "Hashimoto's encephalopathy," has been a subject of interest at our center, ${ }^{3,4}$ but no such case was identified in our search. This may be due to either the age of onset being after age 45 and/ or such patients often being quite ill and hospitalized (again, we purposefully sought to identify patients presenting in the outpatient setting).

One patient was diagnosed with Fahr disease. Interestingly, this patient experienced progressive cognitive decline, but did not exhibit a movement disorder. Although Fahr disease is typically associated with movement disorders, behavioral disturbances and dementia, a recent case report described a patient with Fahr disease who presented with presenile dementia in the absence of substantial movement disorder. ${ }^{5}$

\section{Comparison to clinical data on young onset CJD}

A meta-analysis examining the age dependence of phenotypic presentations of CJD reported differences in the rates of several symptoms when comparing patients having onset before age 50 to those with onset after age 50 . These differences in presentation were noted even when the analysis was restricted to patients having sporadic CJD (sCJD). ${ }^{6}$ Similar findings were reported in a review of young-onset CJD cases (presentation prior to age 30), which suggested that the clinical presentations of younger patients with SCJD more closely resembling variant CJD (vCJD) than the clinical features of SCJD in older patients. ${ }^{7}$

Psychiatric symptoms occur at a much higher frequency in younger individuals who develop CJD. ${ }^{6-8}$ This was previously thought to represent a feature specific to vCJD, ${ }^{9}$ but subsequent work has found this to be true among younger patients with non-variant CJD. ${ }^{6-8}$ This observation held true in our cohort as well, with five of the seven CJD patients exhibiting early affective or behavioral changes. Interestingly, these symptoms were also common among individuals having RP-YOD due to other etiologies. It is unclear whether this finding may be due to observer bias, a greater tendency for younger patients and their families to report psychiatric symptoms, or whether they may represent an age-dependent host response to neurological disease.

Age younger than 50 was also associated with higher likelihood of presenting with cerebellar/ gait disturbance and myoclonus among sCJD patients. ${ }^{6}$ The presenting symptoms of the seven CJD patients included in our cohort did mirror this reported experience, although the frequency of myoclonus (57\%) and cerebellar/gait difficulties (57\%) were much higher than the respective $11.6 \%$ and $29 \%$ reported in that meta-analysis. A review of young-onset CJD reported that $41 \%$ of cases presented with cerebellar syndromes, ${ }^{7}$ which fits well with the high frequency observed in our cohort. Of the seven patients exhibiting myoclonus at presentation, four had CJD and three had other diagnoses, suggesting that the presence of myoclonus in association with RP-YOD has limited utility in predicting etiologic substrate. Parkinsonism 
was noted in only one CJD patient in our cohort (14\%), also in line with the previously reported frequency in younger CJD patients.

\section{Utility of MRI}

As expected, MRI was helpful in narrowing the differential, suggesting a specific diagnosis in about one-third of the cohort. Non-diagnostic abnormalities were identified in most of the other patients. MRI changes characteristic of CJD were identified in about half of the patients having $\mathrm{CJD}$, a somewhat lower rate than is typically reported for $\mathrm{SCJD},{ }^{10}$ but within the range reported in young-onset CJD. ${ }^{8}$ This study of young-onset SCJD included FLAIR in half of the patients and few had DWI for review. However, this study compared a group of older SCJD patients accrued over the same interval, and reported a significantly lower rate of MRI abnormality. It is unclear whether this abnormality would be observed if all patients (both young and old) had FLAIR and DWI sequences as part of their evaluation, and it is also unclear whether this relates to the age-dependent codon 129 genotype difference observed in this study. ${ }^{8}$

\section{Utility of laboratory studies}

Serologic investigation identified one disease (HIV) for which specific treatment was available. It is likely that a strong referral bias effect was present, as patients identified to have a diagnosis, particularly one for which treatment exists, may be unlikely to be referred to a tertiary center for further evaluation. This referral bias likely also resulted in fewer patients experiencing rapidly progressive dementia related to HIV, chemical dependency or other problems more commonly treated in urban settings. Thus, one would expect that serologic studies would have a higher diagnostic yield outside of the context of a tertiary referral center. One patient had paraneoplastic limbic encephalitis and ultimately made dramatic and sustained improvement following treatment of his retroperitoneal teratoma. No specific antibody was identified in serum at the time of that patient's evaluation.

CSF analysis identified an abnormal 14-3-3 protein and/or NSE level in four of five tested CJD patients, which is consistent with the reported utility of CSF in younger patients with SCJD. ${ }^{8}$ Spinal fluid analysis was repeated in many patients within this cohort, usually to followup non-specific CSF abnormalities (e.g. mildly elevated protein) or to obtain additional testing evaluating for a specific diagnosis. In most cases, these repeat CSF analyses did not result in identification of a specific diagnosis.

\section{Utility of EEG}

EEG findings suggested a specific diagnosis in one patient (PSWCs in patient 15, diagnosis CJD). A retrospective analysis of SCJD presenting prior to age 50 reported that PSWCs were identified in only $24 \%,{ }^{8}$ and a literature review of young-onset SCJD reported a similar figure of $26 \%{ }^{7}$ These rates are much lower than those reported in typical older-onset sCJD. In other patients, the presence of only non-specific EEG findings allowed narrowing of the differential diagnosis and decreased the likelihood that subclinical seizures contributed to the clinical presentation. The diagnostic yield of EEG may also be underrepresented in this retrospective cohort who had been referred to a tertiary center for further evaluation, as those in which prior EEGs identified diagnostic abnormalities may not have been referred for further evaluation.

\section{Biopsy}

Cerebral biopsy identified specific pathological findings in two of six patients (33\%), consistent with the rate reported another series investigating the diagnostic yield of biopsy in dementia. ${ }^{11}$ In that series, nonspecific changes were the most common pathological finding, as was the case in our series. Two patients in our series (one with Fahr's disease and one with FTLD-MND) had nondiagnostic biopsy findings but a diagnosis was able to be established at 
autopsy. This represents one-third of the patients who underwent biopsy and nearly $10 \%$ of the cohort as a whole, underscoring the importance of postmortem examination.

\section{Conclusions}

Younger patients presenting with a rapidly progressive dementia appear to have many common clinical features. Affective and behavioral disturbances are frequently identified in these younger patients, as are cerebellar symptoms and visual/occulomotor abnormalities. It is interesting to speculate that these observed similarities might implicate a common neuroanatomical basis despite the varied etiologic causes of RP-YOD in this cohort, although why this should be so remains unclear.

Patients in this age demographic are likely to have dependent children and spouses, and establishing an accurate diagnosis is essential to provide prognostic information, allowing appropriate social and financial counseling. ${ }^{12}$ Siblings or adult children of these patients may be contemplating having children. Identification of a disorder with a genetic or hereditary basis may allow for appropriate genetic counseling (in addition to the genetic counseling which must precede genetic testing). Although often of limited utility when viewed individually, serologic studies, CSF analysis, MRI and EEG data may allow clinicians to arrive at a diagnosis, and in some cases specific treatment may be recommended. Six patients in our cohort had indeterminate etiologies despite extensive antemortem evaluation and, in one case, autopsy. Clearly, our understanding of young-onset dementia is incomplete, and establishing a definitive diagnosis may remain elusive in some patients, despite a thorough evaluation.

\section{Acknowledgments}

This research was supported by National Institute on Aging grants AG06786, AG16574, AG11378 and AG07216, and the Robert H. and Clarice Smith and Abigail Van Buren Alzheimer's Disease Research Program of the Mayo Foundation.

\section{References}

1. Geschwind MD, Haman A, Miller BL. Rapidly progressive dementia. Neurol Clin 2007;25(3):783807. vii. [PubMed: 17659190]

2. Hoffman Snyder C, Mishark KJ, Caviness JN, et al. Nonvasculitic autoimmune inflammatory meningoencephalitis imitating Creutzfeldt-Jakob disease. Arch Neurol 2006;63(5):766-8. [PubMed: 16682549]

3. Castillo P, Woodruff B, Caselli R, et al. Steroid-responsive encephalopathy associated with autoimmune thyroiditis. Arch Neurol 2006;63(2):197-202. [PubMed: 16476807]

4. Sawka AM, Fatourechi V, Boeve BF, Mokri B. Rarity of encephalopathy associated with autoimmune thyroiditis: a case series from Mayo Clinic from 1950 to 1996. Thyroid 2002;12(5):393-8. [PubMed: 12097200]

5. Modrego PJ, Mojonero J, Serrano M, Fayed N. Fahr's syndrome presenting with pure and progressive presenile dementia. Neurol Sci 2005;26(5):367-9. [PubMed: 16388376]

6. Appleby BS, Appleby KK, Rabins PV. Does the presentation of Creutzfeldt-Jakob disease vary by age or presumed etiology? A meta-analysis of the past 10 years. J Neuropsychiatry Clin Neurosci 2007;19 (4):428-35. [PubMed: 18070846]

7. Corato M, Cereda C, Cova E, et al. Young-onset CJD: age and disease phenotype in variant and sporadic forms. Funct Neurol 2006;21(4):211-5. [PubMed: 17367581]

8. Boesenberg C, Schulz-Schaeffer WJ, Meissner B, et al. Clinical course in young patients with sporadic Creutzfeldt-Jakob disease. Ann Neurol 2005;58(4):533-43. [PubMed: 16037975]

9. Zeidler M, Johnstone EC, Bamber RW, et al. New variant Creutzfeldt-Jakob disease: psychiatric features. Lancet 1997;350(9082):908-10. [PubMed: 9314868]

10. Shiga Y, Miyazawa K, Sato S, et al. Diffusion-weighted MRI abnormalities as an early diagnostic marker for Creutzfeldt-Jakob disease. Neurology 2004;63(3):443-9. [PubMed: 15304574] 
11. Warren JD, Schott JM, Fox NC, et al. Brain biopsy in dementia. Brain 2005;128(Pt 9):2016-25. [PubMed: 15901648]

12. Kaiser S, Panegyres PK. The psychosocial impact of young onset dementia on spouses. Am J Alzheimers Dis Other Demen 2006;21(6):398-402. [PubMed: 17267371] 


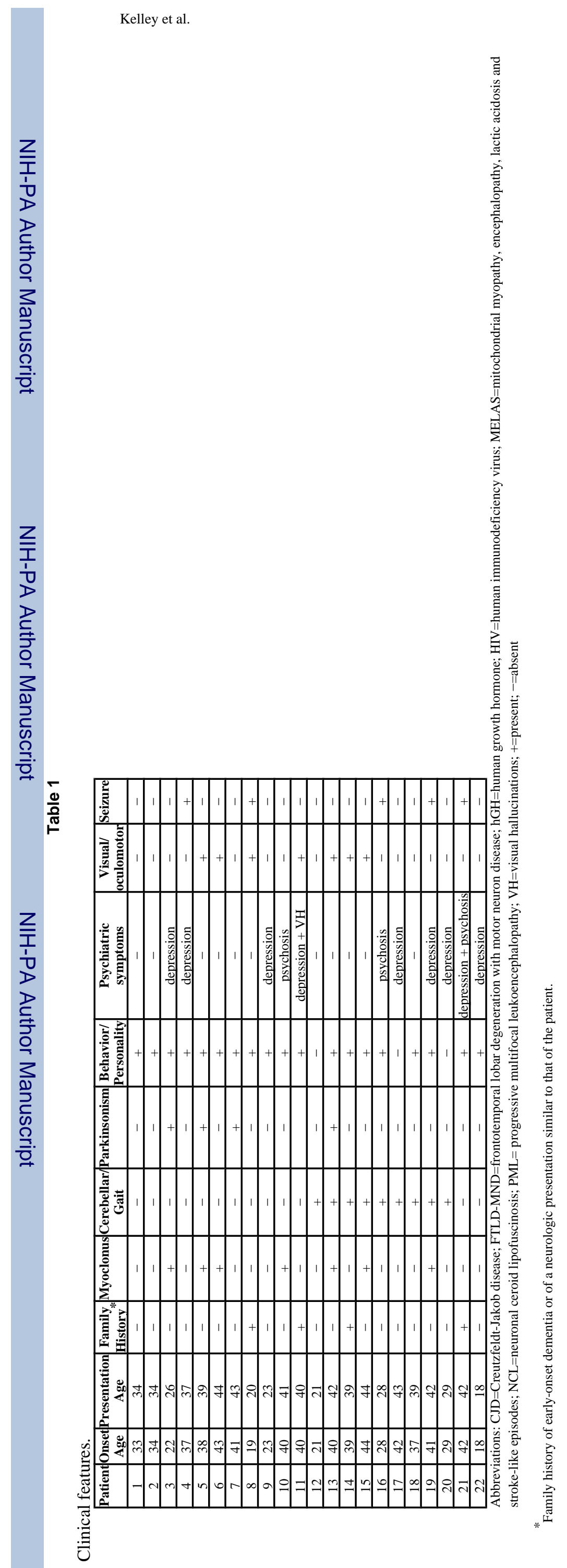



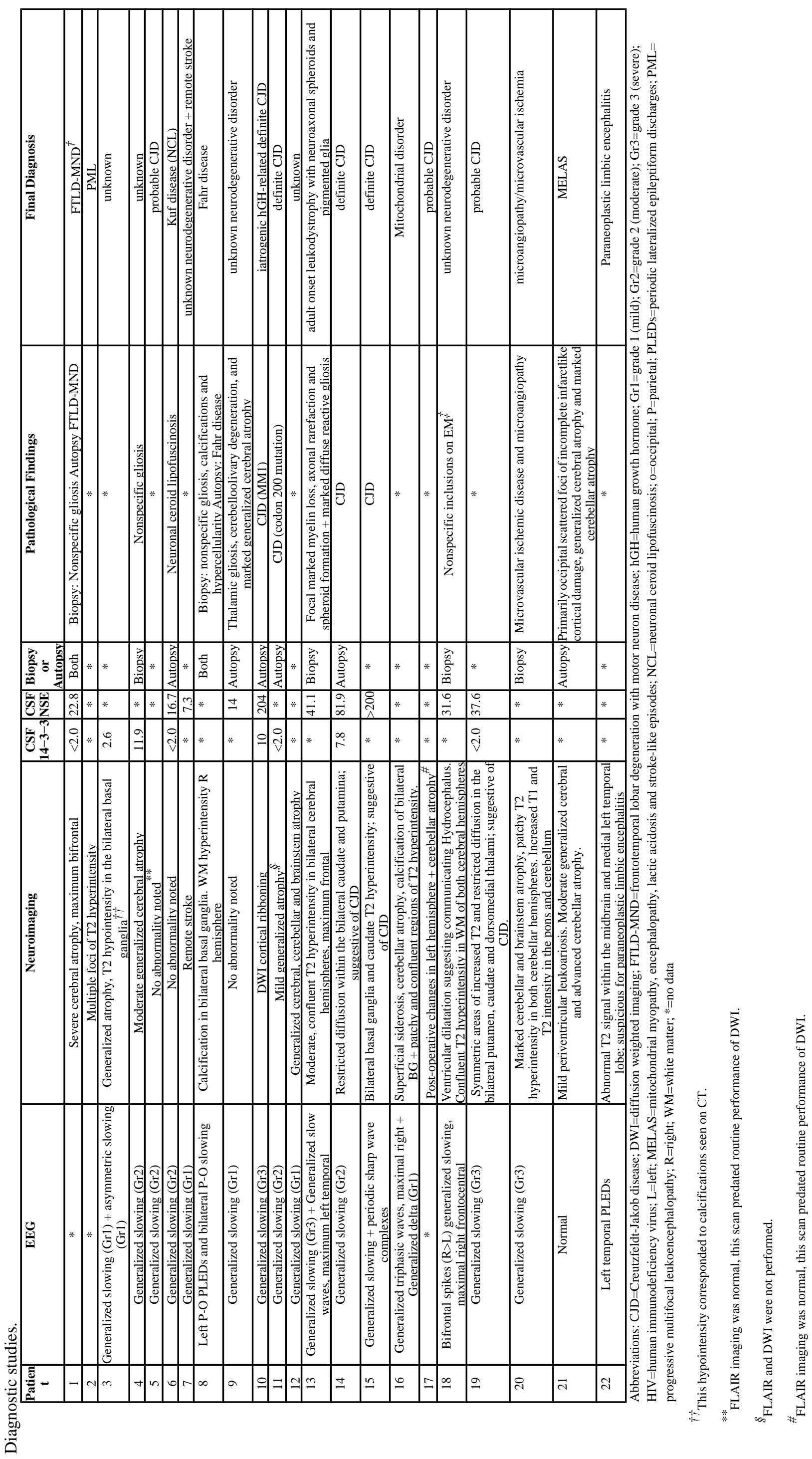
